Utah State University

DigitalCommons@USU

$1-1-2011$

\title{
Preflight Assessment of the Cross-track Infrared Sounder (CrIS) Performance
}

Vladimir V. Zavyalov

Chad S. Fish

Gail E. Bingham

Mark Esplin

Mark Greenman

Deron Scott

See next page for additional authors

Follow this and additional works at: https://digitalcommons.usu.edu/sdl_pubs

\section{Recommended Citation}

Zavyalov, Vladimir V.; Fish, Chad S.; Bingham, Gail E.; Esplin, Mark; Greenman, Mark; Scott, Deron; and Han, Yong, "Preflight Assessment of the Cross-track Infrared Sounder (CrIS) Performance" (2011). Space Dynamics Lab Publications. Paper 152.

https://digitalcommons.usu.edu/sdl_pubs/152

This Article is brought to you for free and open access by the Space Dynamics Lab at DigitalCommons@USU. It has been accepted for inclusion in Space Dynamics Lab Publications by an authorized administrator of DigitalCommons@USU. For more information, please contact digitalcommons@usu.edu.

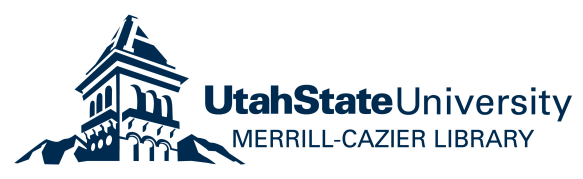


Authors

Vladimir V. Zavyalov, Chad S. Fish, Gail E. Bingham, Mark Esplin, Mark Greenman, Deron Scott, and Yong Han 


\title{
Preflight assessment of the Cross-track Infrared Sounder (CrIS) performance
}

\author{
Vladimir V. Zavyalov", Chad S. Fish ${ }^{\mathrm{a}}$, Gail E. Bingham ${ }^{\mathrm{a}}$, Mark Esplin ${ }^{\mathrm{a}}$, Mark Greenman ${ }^{\mathrm{a}}$, \\ Deron Scott ${ }^{\mathrm{a}}$, and Yong Han ${ }^{\mathrm{b}}$ \\ ${ }^{a}$ Utah State University/Space Dynamics Laboratory, 1695 North Research Park Way, \\ North Logan, Utah 84341 \\ ${ }^{\mathrm{b}}$ National Oceanic and Atmospheric Administration, National Environmental Satellite Data and \\ Information Service, Center for Satellite Applications and Research, \\ 5200 Auth Road, Camp Springs, MD
}

\begin{abstract}
The Cross-track Infrared Sounder (CrIS) is a part of the Crosstrack Infrared and Microwave Sounding Suite (CrIMSS) that will be used to produce accurate temperature, water vapor, and pressure profiles on the NPOESS Preparatory Project (NPP) and upcoming Joint Polar Satellite System (JPSS) operational missions. The NPP CrIS flight model has completed sensor qualification, characterization, and calibration and is now integrated with the NPP spacecraft in preparation for the launch. This paper reviews the CrIS performance during thermal vacuum tests, including the spacecraft integration test, and provides a comparison to the AIRS and IASI heritage sensors that it builds upon. The CrIS system consists of the instrument itself and ground-based scientific algorithms. The data reported in this paper was processed with the latest version of the CrIS science sensor data record (SDR) algorithm and thus reflects the performance of the CrIS SDR system. This paper includes the key test results for Noise Equivalent Differential Noise $(\mathrm{NEdN})$, Radiometric Performance, and Spectral Accuracy. The CrIS sensor performance is outstanding and will meet the mission needs for the NPP /JPSS mission. NEdN is one of the key performance tests for the CrIS sensor. The overall NEdN performance for the CrIS in the LWIR, MWIR and SWIR spectral bands is excellent and is comparable or exceeds NEdN performance of AIRS and IASI. Also discussed is the Principal Component Analysis (PCA) approach developed to estimate contribution of random and spectrally correlated noise components to the total NEDN.
\end{abstract}

Keywords: CrIS, ATMS, NPOESS Preparatory Project, JPSS, Calibration, Validation

\section{INTRODUCTION.}

The Joint Polar Satellite System (JPSS) mission is a joint effort involving the National Aeronautics and Space Administration (NASA) and National Oceanographic and Atmospheric Administration (NOAA). This satellite system developed earlier as the U.S. National Polar-orbiting Operational Environmental Satellite System (NPOESS) is being developed to monitor global environmental conditions and collect and disseminate data related to weather, atmosphere, oceans, land, and near-space environment. The JPSS mission is currently scheduled to launch in October 2011.

The Cross-track Infrared Sounder (CrIS) is a key component of the larger Cross-track Infrared/Microwave Sounding Suite (CrIMSS), which consists of the CrIS and the Advanced Technology Microwave Sounder (ATMS) as illustrated in Figure 1. Together, these instruments provide precise measurements of vertical distributions of temperature, moisture, and pressure within the Earth's atmosphere. CrIS is a sophisticated interferometric sounding sensor which accurately measures upwelling infrared radiance at very high spectral resolution ${ }^{[1]}$. The science heritage for CrIS was derived from the Atmospheric Infrared Radiation Sounder (AIRS) on the NASA EOS Aqua satellite ${ }^{[2]}$ and Infrared Atmospheric Sounding Interferometer (IASI) on the European Metop platform ${ }^{[3] ~[4]}$.

The data will be used to significantly improve the accuracy and timeliness of warnings for severe weather events both nationally and worldwide. It is expected that the impact on weather forecasting of the single CrIS on NPP will be similar to what has been demonstrated with AIRS and IASI. The NPP CrIS flight model (FM1) has completed sensor qualification, characterization, and calibration and is now integrated with the NPP spacecraft in preparation for the launch.

Sensors, Systems, and Next-Generation Satellites XV, edited by Roland Meynart, Steven P. Neeck, Haruhisa Shimoda, Proc. of SPIE Vol. 8176, 817606 · (c) 2011 SPIE · CCC code: 0277-786X/11/\$18 · doi: 10.1117/12.897674 
The CrIS system is defined as a space born instrument and ground based scientific algorithms. The CrIS observations data is delivered to the users in the form of the Raw Data Record (RDR), Sensor Data Records (SDR), and

Environmental Data Records (EDRs) as schematically depicted in Figure 1. The level 1B ground segment algorithm transforms raw instrument measurements (RDR product produced by the on-board software) to the geo-located spectrally and radiometrically calibrated spectra (SDR product). The SDRs are subsequently used by the separate EDR algorithm to retrieve atmospheric temperature, moisture, and pressure profiles forming EDR product.

This paper describes the key parameters of the CrIS SDR product performance including Noise Equivalent differential Noise (NEdN), Radiometric Performance, and Spectral Accuracy based on the ground test results. All the results reported herein were processed with the latest version of the CrIS SDR algorithm and thus the performance of the entire CrIS system (instrument and SDR algorithm) has been tested and the results are included in this paper.

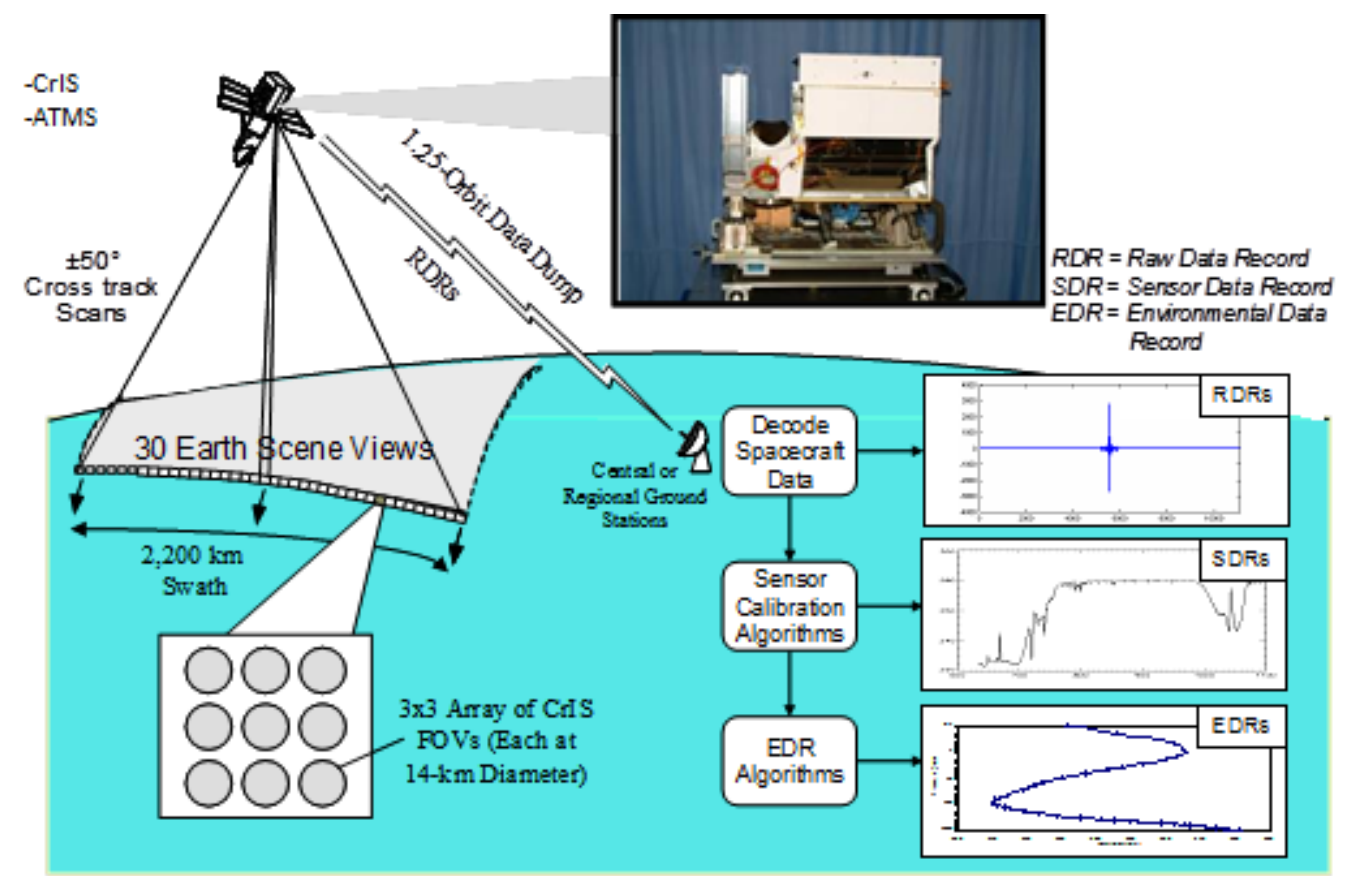

Figure 1. CrIS diagram showing concept of the CrIS measurements.

\section{CrIS SYSTEM DESCRIPTION}

CrIS is a Fourier Transform Spectrometer (FTS) built by ITT Industries, Space Systems Division. The instrument has 1305 channels in three spectral bands from $650-2155 \mathrm{~cm}^{-1}$, with spectral resolutions of $0.625 \mathrm{~cm}^{-1}, 1.25 \mathrm{~cm}^{-1}$, and $2.5 \mathrm{~cm}^{-1}$ in the long-wave (LWIR), mid-wave (MWIR), and short-wave (SWIR) bands respectively. The cross-track scan spans 49.395 degrees on either side of the nadir and consists of a total of 30 earth view positions, or Fields of Regard (FOR) as schematically shown in Figure 1. The CrIS focal plane has nine detectors arranged as a 3x3 array and simultaneously produces radiometric measurements over the nine Fields of View (FOV) at each scan position.

The CrIS instrument has a number of design features which allow it to achieve high performance in a relatively small volume. A compact optical design provides a large 8-cm aperture for superior sensitivity. An innovative four-stage passive cooler provides low-temperature detector operation without the complexities of an active cooler. Photovoltaic detectors are used in all three spectral bands for maximum sensitivity and linearity. A 3x3 array of photo-detectors (each with a $14 \mathrm{~km}$ diameter at nadir) is used in each spectral band for improved spatial coverage. The interferometer uses a proven plane-mirror, Michelson design with dynamic alignment, to maintain precise alignments between the two arms of the interferometer. A deep-cavity Internal Calibration Target (ICT) and deep space view (DS) are used for improved calibration accuracy performance. Finally, a passive vibration-isolation system enables CrIS to operate on spacecraft with relatively high level of disturbances. Other parameters of the CrIS instruments are shown in Table 1 in comparison with AIRS and IASI sounding instruments. 
Table 1. Comparison of CrIS main parameters to AIRS and IASI instruments

\begin{tabular}{|c|c|c|c|}
\hline & CrIS (on NPP) & AIRS (on EOS Aqua) & IASI (on MetOp) \\
\hline Weight (kg) & 147 & 177 & 236 \\
\hline Size (cmxcmxcm) & $80 \times 47 \times 66$ & $116.5 \times 80 \times 95.3$ & $120 \times 110 \times 110$ \\
\hline Power (Watts) & 110 & 220 & 210 \\
\hline Data Rate (Mbits/sec) & 1.44 & 1.28 & 1.5 \\
\hline
\end{tabular}

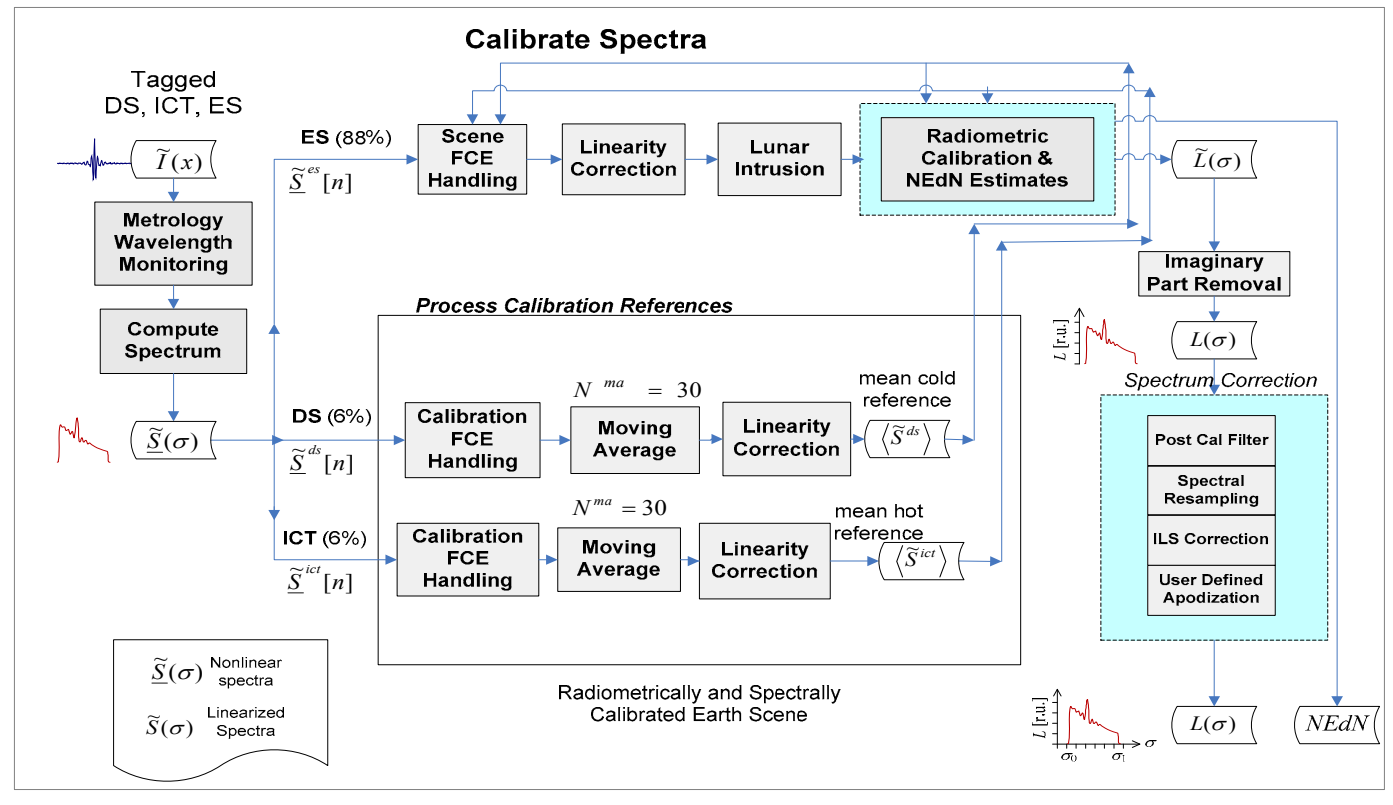

Figure 2. Ground processing SDR algorithm data flow

The CrIS SDR algorithm allows for accurate spectral and radiometric calibration of measured upwelling radiances. The flow chart of the CrIS SDR algorithm is shown in Figure 2. The major steps performed by the CrIS SDR code are as follows: unpack data and remove compression (bit trim); perform laser metrology spectral calibration using neon reference (once per orbit); assess data quality; perform a discrete Fourier transform; detect and correct fringe count errors (rare); correct for non-linearity; perform moving window average of the DS (cold) and the ICT (warm); apply two-point radiometric calibration in complex radiance space; calculate ICT radiance and correct for environment (reflected energy); remove background offset and phase dispersion; apply post-calibration filter; resample spectral channel centers to a fixed grid; perform instrument line shape (ILS) correction; compute line of sight relative to the spacecraft body frame for geolocation calculation; and format output. The detailed description of the SDR algorithm theoretical basics and algorithm implementation can be found in the CrIS ATBD document ${ }^{[5]}$.

\section{CrIS FM1 TEST RESULTS}

The CrIS team has successfully completed the FM1 test program and has shown that overall sensor performance is outstanding. The CrIS instrument has completed numerous tests including the following:

\section{Test}

- Extensive module testing and instrument integration

- Bench testing; EMI testing

- Vibration testing

- Post vibe bench testing

\section{Results}

Good performance

Good instrument performance

Excellent modal response repeatability

Excellent detector registration 


\section{Test}

- $\quad$ Final Thermal Vacuum Testing (TVAC) performed in several stages (TVAC1-TVAC4)

- $\quad$ Short term repeatability and long term repeatability in TVAC

- Electrical performance testing

- FOV shape/co-registration

\section{Results}

Excellent radiometric, spectral, and -noise Performance demonstrated Meet program requirements

Meet program requirements Excellent stability and meets FOV requirements

Additionally the CrIS sensor has been subjected to the TVAC test after being integrated to the spacecraft (S/C TVAC). During S/C TVAC test mostly noise performance of the instrument was monitored. This paper will primarily focus on reporting the CrIS FM1 testing results in final TVAC testing under the mission nominal conditions. In addition to Mission Nominal, the CrIS instrument has been conservatively tested at Mission High, Mission Low, and Survival Low conditions. The Mission Nominal conditions best represent the on-orbit environment and the results reported here represent an expected system performance on orbit.

All results reported in this paper were obtained by processing CrIS raw interferograms (RDR product) with CrIS science grade SDR algorithm.

\subsection{Noise performance}

$\mathrm{NEdN}$ is one of the key performance tests for the CrIS sensor characterizing end-to-end performance of the instrument. The overall NEdN performance of the CrIS instrument is excellent in all spectral bands (LWIR, MWIR and SWIR) and exceeds specification requirements.

The mission nominal results acquired during the TVAC4 test are shown in Figure 3 (a) where each of the plots show measured NEdN for all nine FOVs at a scene temperature of $287 \mathrm{~K}$ (instrument is looking at the external calibration target ECT at specified temperature). In Figure 3 (b) the NEdN performance observed during the S/C TVAC integration test is shown. It is clear that the noise level is much better than the specification requirements and is very stable in all spectral bands for most of the FOVs during the series of CrIS ground tests.

During various CrIS tests (bench and TVAC1-TVAC4), the change in the MWIR NEdN level has been observed for several MWIR FOVs $(2,3,7$, and 8). Figure 3 demonstrates the out-of-family NEdN level observed for MWIR FOV7 during the latest TVAC4 and S/C TVAC tests. During TVAC3, the NEdN level of MWIR7 was lower than during TVAC4 and in a family with other FOVs as shown in Figure 4 (a). The root cause of this behavior was isolated to the detector diode material that changes parameters with warm-up/cool-down cycles.
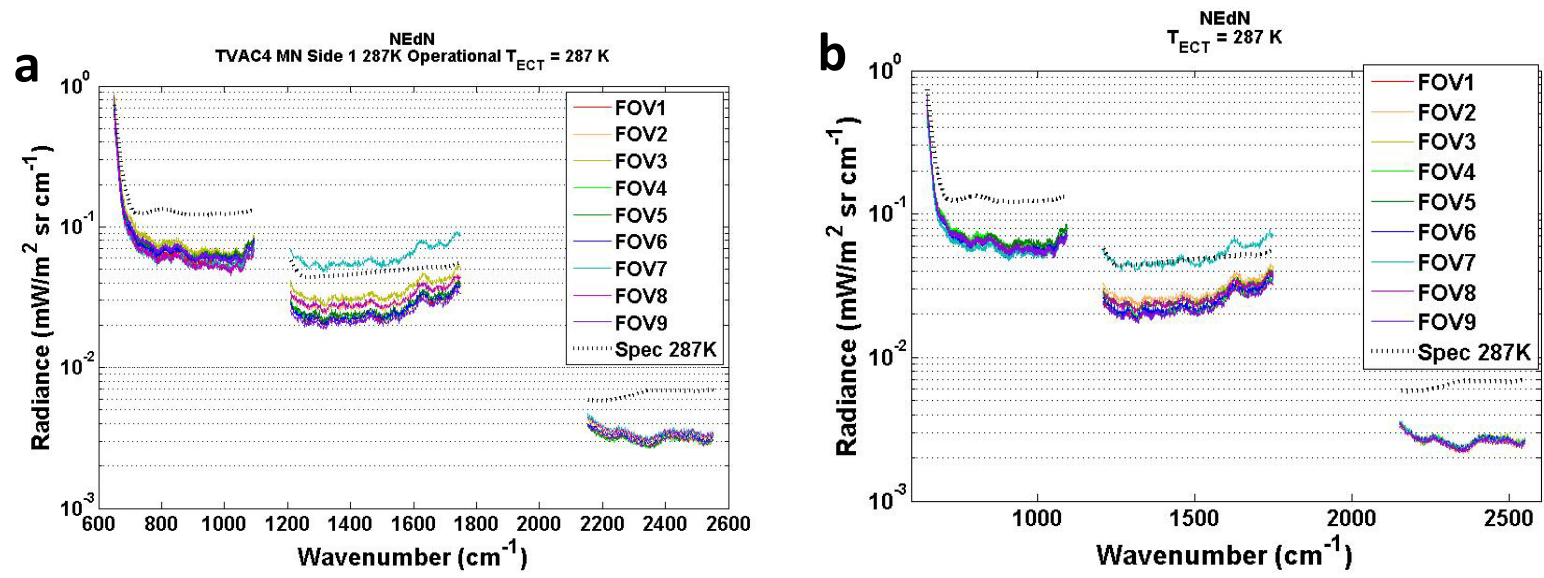

Figure 3. Nominal CrIS NEdN performance during TVAC4 (a) and S/C integration TVAC (b) tests.

The PCA analysis of the correlated and random components of the NEdN (see details in the Appendix) confirmed that the increase in the NEdN of MWIR FOV7 is due to the random noise component that is consistent with additional noise after photo diode parameters change. The results of PCA analysis of MWIR FOV7 NEdN observed during TVAC3 and 
TVAC4 tests are shown in Figure 4 (a) and (b) respectively. In both cases, a random noise component dominates total NEdN.
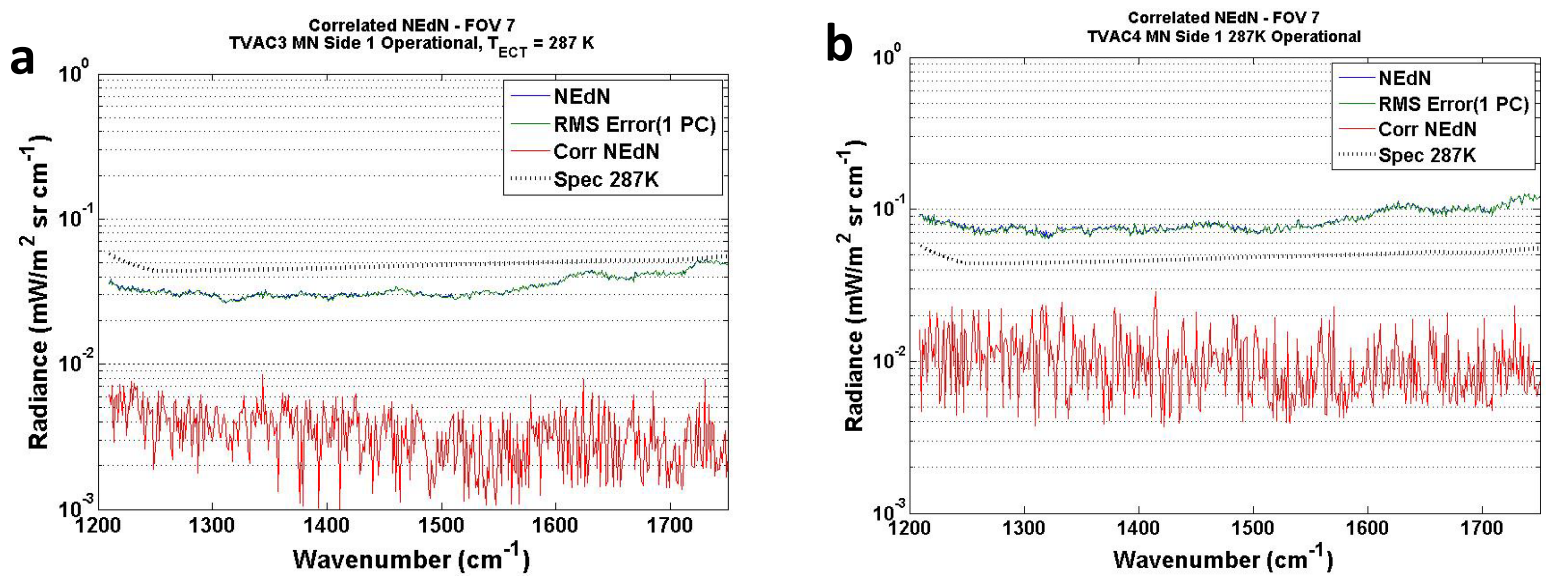

Figure 4. Contribution of random (green) and correlated (red) noise components to the total NEdN (blue) in the MWIR FOV7 estimated during TVAC3 (a) and TVAC4 (b) tests.

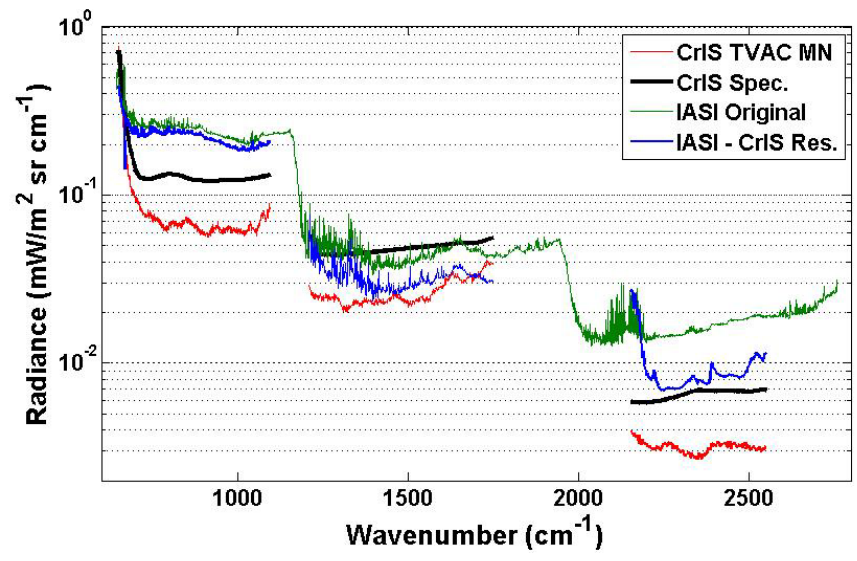

Figure 5. Comparison of CrIS TVAC measured nominal noise (red) with IASI NEdN estimated at IASI native (green) and CrISlike (blue) spectral resolutions. The black curve depicts CrIS spec requirements.

In-flight NEdN performance of the CrIS instrument is expected to be as shown in Figure 3. In Figure 5, the CrIS NEdN observed during TVAC tests (red curve) is compared with IASI NEdN estimated from the IASI on-orbit observations (green curve) using the PCA approach for atmospheric spectra ${ }^{[8]}$. The same IASI spectra were resampled to the CrIS spectral resolution and spectral band coverage using our Fourier transform algorithm. This algorithm is based on the truncation of the IASI full resolution interferograms to the CrIS sampling intervals in the interferogram domain. These spectra were then subjected to the PCA analysis (the same as original IASI spectra) to estimate IASI noise at CrIS spectral resolution and band coverage. The resulting IASI NEdN at CrIS spectral resolution is also plotted in Figure 5 (blue curve). It is clear that the expected NEdN performance of the CrIS instrument is comparable or better (in LWIR and SWIR spectral bands) than the IASI instrument noise even at CrIS spectral resolution.

Based on this data, the following conclusions pertaining NEdN performance of the CrIS instrument can be made:

- NEdN estimated during different TVAC tests including S/C integration test is very consistent and stable.

- Overall TVAC NEdN performance is outstanding and meets the specification limits in all spectral bins and under nearly all test conditions.

- CrIS NEdN is comparable with or better than NEdN level of heritage AIRS and IASI sensors.

- Minor NEdN outages in several spectral bins have been observed and are well understood. These outages have negligible SDR/EDR mission performance impact. 
- Larger outages were observed in the MWIR band for several FOVs (FOV 2, 3, 7, and 8). The change in NEdN is determined mostly by the random noise component.

- Continuous degradation of NEdN was not observed during TVAC, once cool-down was completed detectors remain stable.

- The root cause was isolated to the MCT detector diode material that changes parameters with warm-up/cooldown cycles. Migrating impurities in the IR diode interfaces can create/change trap states during warm-up/cooldown cycles.

- As a rule change in NEdN correlates with changes in non-linearity (NL) coefficients (see paragraph 3.2).

- Possible change in NEdN and NL coefficients is expected in the MWIR spectral band after launch.

\subsection{Radiometric performance}

The CrIS sensor accurately measures the spectral radiance of earth scenes. Radiometric calibration of the sensor is a process of assigning absolute value in radiance units to the intensity of acquired spectra with a specified accuracy. As a part of the radiometric calibration process, the CrIS sensor uses a deep cavity onboard blackbody calibration target (ICT) that emits a known radiance for a given temperature and DS view as standard reference sources. The main contributors to the CrIS radiometric uncertainty are ICT temperature uncertainty, ICT emissivity knowledge, and residual non-linearity correction errors. The roll up of the major uncertainties contributing to the total radiometric uncertainty (RU) of the instrument is shown in Figure 6. The analysis has been performed by the ITT team and contributing uncertainties were deducted from the design analysis, test data including TVAC, and operational information.

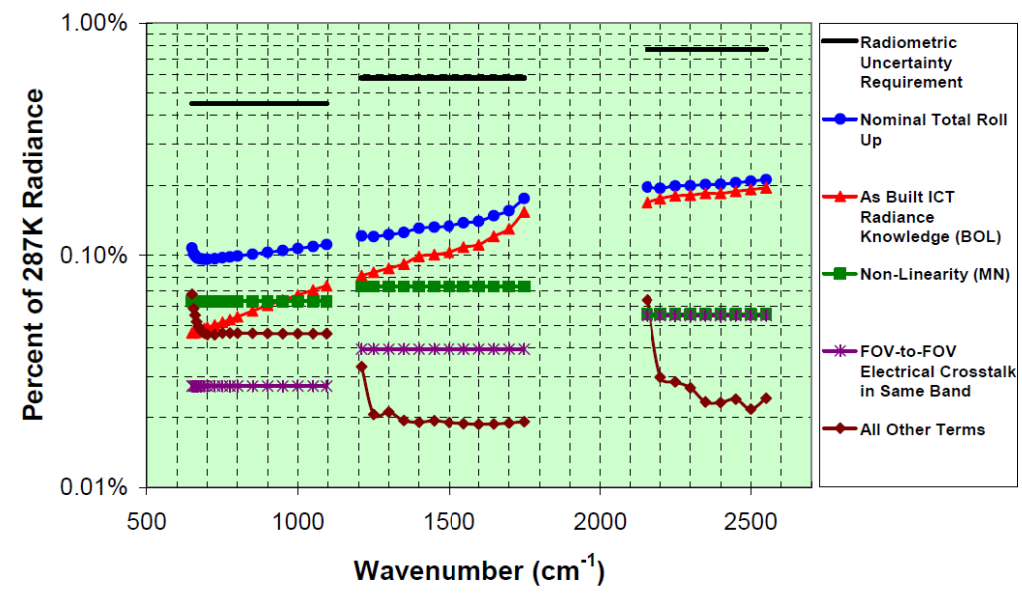

Figure 6. CrIS nominal radiometric uncertainty performance built as a roll-up of the major uncertainties.

The temperature of the ICT is monitored with NIST traceable PRT's that are stable over the CrIS mission life. The ICT emissivity was retrieved from the TVAC3 data set where the temperatures of the ECT and ICT were set to the same value at $315 \mathrm{~K}$. Independent emissivity test using ITT NIST traceable Transfer Standard Stability Radiometer was also conducted and the results were used as an anchor point for the retrievals to further improve the emissivity knowledge accuracy.

The FOV to FOV electrical cross-talk is a top-level rollup term where the worst case for each band was estimated. It uses TVAC2 mission nominal data acquired during FOV slit scan (one row or column of FOVs is illuminated by slit scan) evaluating amount and effect of the electrical cross-talk for each FOV.

Most of CrIS spectral bands have some level of non-linearity (NL) that contributes to the overall RU. In the LWIR and MWIR bands, the detectors have an inherent NL which is large enough to require a correction implemented in the ground SDR algorithm. NL in the SWIR band is negligible small and no correction is required. The correction approach is a simple quadratic correction based on the heritage of a University of Wisconsin (UW) technique ${ }^{[6]}$. This technique uses a quadratic correction term (referred as to ' $\mathrm{a}_{2}$ ' coefficient) and telemetry data provide information on the background instrument flux (voltage offset). The residual error after NL correction produces a radiometric calibration error taken into account in the final RU rollup. 
As in the case of NEdN discussed above, the NL characteristics of the LWIR and MWIR detectors can shift after the detectors has been warmed up to ambient and cooled back down to their cryogenic operating temperature. This is particularly true for most non-linear MWIR detectors such as MWIR 2, 7, and 8. This has happened twice during ground tests TVAC3 and TVAC4 after the warm-up and cool-down cycles. It is important to note that when the temperature of the detectors reaches their cryogenic operating temperature, no NL change was observed and NL parameters were stable for both spectral bands. The same behavior is expected on orbit.

To account for the change in NL coefficients, the NL adjustment methodology has been developed. This technique is based on the analysis of out-of-band response of the magnitude spectra taken in diagnostic mode. The out-of-band spectral response observed for MWIR FOV7 during TVAC3 and TVAC4 tests are shown in Figure 7.

These spectra have not been radiometrically calibrated so the vertical scale is relative amplitude; however, the spectra have been scaled so that the in-band spectra have the same maximum amplitude. Diagnostic mode interferograms are necessary for this analysis since for normal mode interferograms, out-of-band energy is filtered out before the data enters the telemetry stream. The effect from quadratic nonlinearity will appear at double the fundamental frequency and also near the zero frequency which is typically used for the NL parameters retrievals. These retrievals are based on the slope of out-of-band low frequency response (see Figure 7).

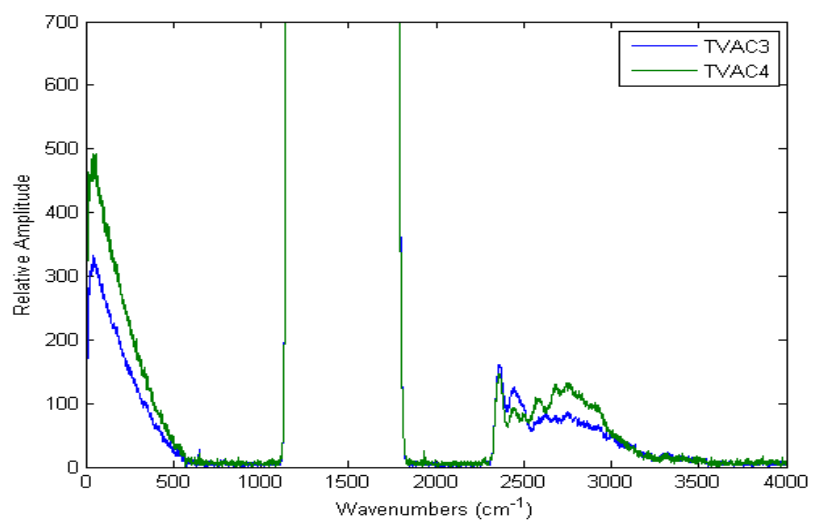

Figure 7. Out-of-band diagnostic mode magnitude spectra for MWIR FOV7.

This 'quadratic' method works perfectly for the LWIR spectral band while MWIR $\mathrm{a}_{2}$ s need further adjustment. This adjustment is based on the fact that FOV6 and FOV9 MWIR detectors have proven to be nearly perfectly linear. In this approach ('empirical' tuning method), the $\mathrm{a}_{2}$ value for each of other seven non-linear detectors is individually adjusted so that the radiometric error matches those of FOV6 and FOV9 on a band averaged basis.

In order to achieve the optimal on-orbit performance, it is necessary to make adjustments to $\mathrm{a}_{2} \mathrm{~s}$ coefficients in orbit as well. Based on the ground test results, a two-stage process for updating $\mathrm{a}_{2}$ in orbit is planned:

- As a first approximation, diagnostic mode interferograms can be used to upgrade LWIR and MWIR $\mathrm{a}_{2}$ coefficients using the 'quadratic' approach developed by the UW and SDL.

- On a second stage, when statistically valuable data on the homogeneous FOR is acquired, the ITT 'empirical method' based on the correlation with linear FOV6 and 9 can be applied to refine the MWIR $\mathrm{a}_{2} \mathrm{~S}$.

The purpose of the TVAC radiometric uncertainty test is to provide additional information that shows that the roll-up values are reasonable. The validation of radiometric calibration of the CrIS (RU estimation) is done by comparing the output radiance of a ground test ECT (with effective emissivity of 0.9995 ) as measured by the CrIS sensor to the calculated output ECT radiances. If the two values agree within the combined uncertainties of the CrIS instrument and the ECT, then there is high confidence in meeting the radiometric uncertainty requirements. For the CrIS sensor, the radiometric uncertainty requirements were evaluated in the 233 to $287 \mathrm{~K} \mathrm{ECT}$ temperature range. In Figure 8 , the RU estimated during TVAC3 and TVAC4 tests is shown at mission nominal conditions and a scene (ECT) temperature of $287 \mathrm{~K}$. The TVAC data was processed with the optimal set of $\mathrm{a}_{2}$ coefficients updated for each test separately. ECT emissivity and reflected term were taken into account during RU estimation. The NL parameters of FOV2 and FOV7 detectors have changed between these two tests; however, the updated $a_{2}$ coefficients for each test produce practically the 
same RU for all FOV including MWIR FOV 2 and 7. It should be noted that the $\mathrm{a}_{2}$ coefficients derived from TVAC4 data were stable and practically did not change between the TVAC4 and S/C TVAC tests.
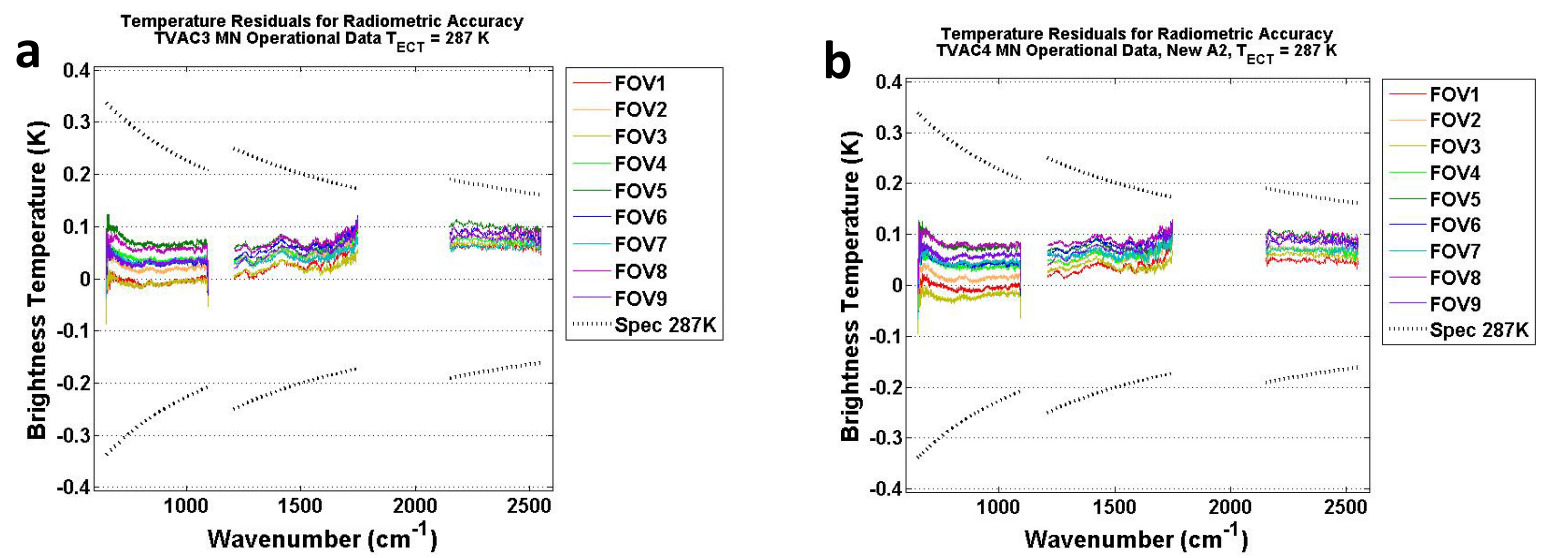

Figure 8. Radiometric uncertainty derived from TVAC3 (a) and TVAC4 (b) data at MN and ECT temperature of $287 \mathrm{~K}$.

In general, the CrIS sensor radiometric test results are outstanding and the performance at the mission nominal conditions meets specifications with margin (see Figure 8). Based on these results, in-flight radiometric uncertainty is expected to be less than $100 \mathrm{mK}$ (one-sigma) and better. Dave Tobin from UW conducted independent estimation of the predicted in-flight radiometric accuracy of the CrIS instrument based on the ground tests results ${ }^{[7]}$. Estimation of uncertainties included ICT temperature and emissivity, components temperature knowledge (frame, optical mechanical assembly, beam splitter, scan baffle), and NL. He concluded that the in-flight RU of CrIS FM1 is estimated to be very good with 3-sigma RU estimates below $200 \mathrm{mK}$ for the large majority of FOVs and spectral channels.

\subsection{Spectral accuracy performance}

Spectral calibration is a process of assigning absolute spectral values to the wavenumber axis (spectral bins). The CrIS system determines the number of half-wavelength zero crossing based on the wavelength of the metrology laser. These zero crossings are used to trigger the sampling of the interferogram. The actual output wavelength of the laser diode is determined by the temperature and current of this device which is monitored to assure that it operates in a single mode. The wavelength metrology system provides laser wavelength measurements based on the comparison with filtered spectral lines from the neon calibration lamp injected into the interferometer with the same optical path as the metrology laser.

The spectral calibration process has to be done with a specified accuracy (less than 10ppm) for all spectral bins and detectors (FOVs). The SDR algorithm provides three major operations: 1) assigns correct wavenumbers to each spectral bin based on the neon lamp reference, 2) resamples the spectral scale on a predefined common spectral axis, and 3) provides ILS correction bringing each spectral bin for all FOVs in its correct position. The SDR algorithm spectral calibration process begins with quality check, unpacking data, and computing laser metrology wavelength from the neon lamp reference source. In orbit, the neon calibration procedure is scheduled to be done at least once per orbit.

The spectral uncertainties rollup has been done by the ITT team and the top level rollup is shown in the Figure 9. Low level spectral uncertainties include laser wavelength drift, off axis FOV location, finite optical pass difference sampling, interferometer alignment errors, apodization effects, aliasing, sampling errors, and detector and amplifier noise. As in the case of RU, contributing spectral uncertainties were deducted from the design analysis, test data, and operational information. It is clear in Figure 9 that total spectral uncertainty of the CrIS system after SDR spectral calibration does not exceed 8ppm for the worst detector (FOV). 


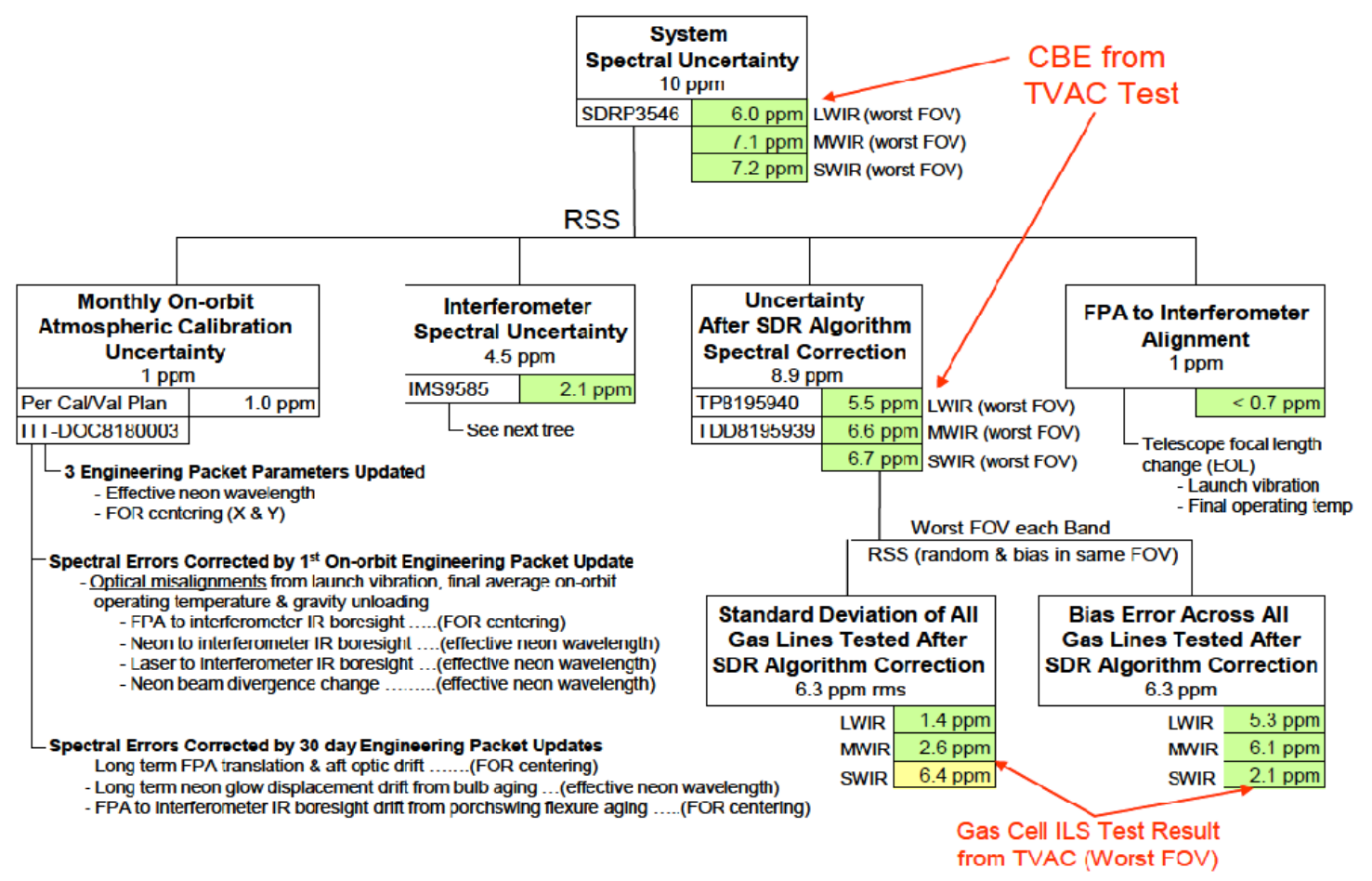

Figure 9. Top level spectral uncertainty rollup.

The spectral uncertainty of the CrIS sensor was verified by viewing gases with known spectral absorption lines. The CrIS sensor views a gas cell that is filled with a gas of known temperature and pressure. The LWIR band is characterized with $\mathrm{CO}_{2}$ gas at 100 Torr. An example of this spectrum is shown in Figure 10 for the LWIR spectral band. The calibrated spectra for all nine FOV's overlay cleanly and correlate to the ideal spectra calculated using line-by-line (LBL) radiative transfer model. The SDR algorithm produces identical ILS in all nine FOVs and successfully removes self-apodization effects aligning all off-axis FOVs to the central one. The MWIR spectral band is characterized with $\mathrm{CH}_{4}$ gas at 40 Torr, and excellent correlation is achieved as in LWIR case. The SWIR is characterized with HBr gas at 175 Torr, and excellent correlation is also achieved.

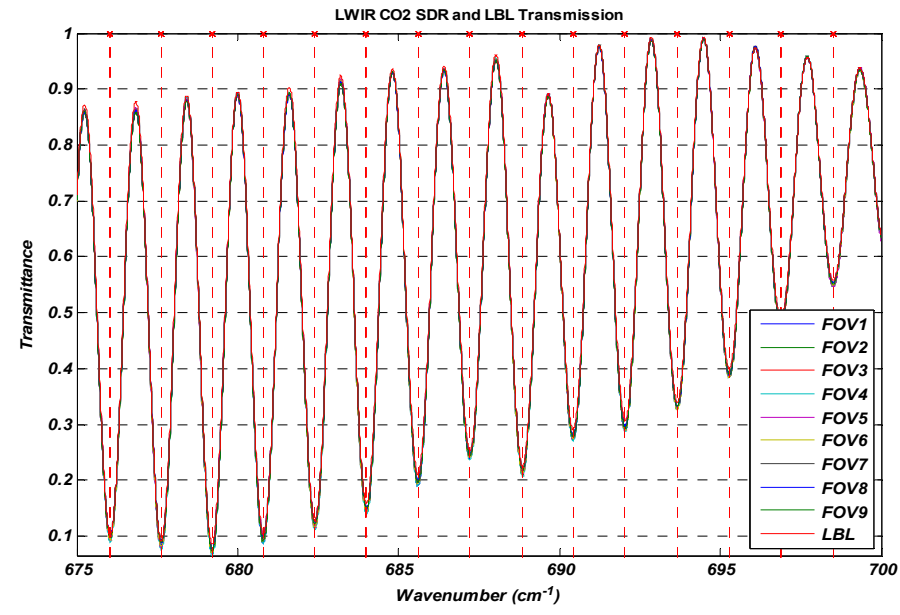

Figure 10. LWIR absorption lines measured with $\mathrm{CO}_{2}$ gas cell for all nine FOVs. Line-by-line (LBL) model is used for theoretical calculations. 
The ability to accurately report spectral line centers observed during gas cell tests is a key ILS performance parameter. The requirement for the CrIS flight unit is that the spectral uncertainty be $<10 \mathrm{ppm}$. The spectral uncertainty as measured for the CrIS FM1 meets the requirements with a large margin of $40 \%$ in LWIR, 29\% in MWIR, and 28\% in SWIR.

ILS shape uncertainty and error was also estimated via simulations supported by the laser ILS test data. The overall ILS shape uncertainties are estimated as follows:

ILS Shape Uncertainty

- LWIR: $0.16 \%(89 \%$ margin $)$

- MWIR: $0.15 \%(90 \%$ margin)

- SWIR: $0.44 \%(71 \%$ margin)

Error with respect to a model for the ILS shape:

- LWIR: $0.1 \%(67 \%$ margin $)$

- MWIR: $0.1 \%(67 \%$ margin $)$

- SWIR: $0.26 \%(13 \%$ margin $)$

The spectral accuracy of the CrIS FM1 is exceptional and the resulting calibrated spectra matched the theoretical spectra extremely well. The overall spectral errors of the CrIS system exceed the specified requirements with a margin.

\section{SUMMARY}

The CrIS FM1 instrument has successfully completed comprehensive performance testing and is now in the process of spacecraft integration. The CrIS system (instrument and SDR ground algorithm) performance is outstanding and will meet the mission needs for the NPP /JPSS mission. The NEdN performance of the CrIS instrument is comparable or better than heritage AIRS and IASI instruments noise performance. The absolute calibration of CrIS radiances in orbit is expected to be better than $0.1 \mathrm{~K}$ of brightness temperature (1-sigma) for all CrIS spectral bands and all scene temperatures. TVAC testing validated this expectation except for scene temperatures lower $250 \mathrm{~K}$ where the external space target had larger emissivity uncertainty. The CrIS system exhibits outstanding spectral performance and exceeds the specification requirements of $10 \mathrm{ppm}$ with a margin. The CrIS sensor will provide the user community with significant new capabilities for atmospheric soundings. Experience with existing hyperspectral sounders like AIRS and IASI provides information on Calibration/Validation efforts appropriate for CrIS ${ }^{[2-4,10]}$. A complimentary set of postlaunch radiance validation techniques, including collocated AIRS /IASI and high altitude aircraft under flights to evaluate the CrIS observed spectra at the $\sim 0.1 \mathrm{~K}$ level, will be included in the NPP/JPSS Cal/Val efforts.

\section{APPENDIX \\ PCA APPROACH FOR INSTRUMENT NOISE CHARACTERIZATION}

Noise of the high resolution spectral instruments can be either correlated or uncorrelated (random) in the spectral domain. The spectrally correlated noise can be found in FTS instruments when the vibration-induced errors caused by uncompensated Michelson mirror tilts are present. Principal Component Analysis (PCA) is a powerful tool for analyzing data from high spectral resolution observations and for redundant data compression ${ }^{[8]}$. On the other hand PCA can be used not only for noise filtering and lossy compression, but also for an independent characterization of sensor noise and other variable instrument artifacts ${ }^{[9]}$.

Here we present our approach for the estimation of random and correlated noise components using PCA techniques in application to the measured set of blackbody (BB) spectra. Estimation of the spectrally correlated $\left(\mathrm{NEdN}_{\mathrm{c}}\right)$ and random components $\left(\mathrm{NEdN}_{\mathrm{r}}\right)$ of the total instrument noise $\left(\mathrm{NEdN}_{t}\right)$ can be obtained from the flight or test data by analyzing the calibrated spectra of onboard calibration BB (ICT) and/or DS view during flight and ECT during ground-based instrument test.

The total BB spectra noise variance can be presented as a sum of random and spectrally correlated noise components ${ }^{[8]}$.

$$
N E d N_{t}^{2}=N E d N_{r}^{2}+N E d N_{c}^{2}
$$

The total instrument noise $\mathrm{NEdN}_{\mathrm{t}}$ is estimated by a standard procedure as the standard deviation of a set of calibrated BB spectra with respect to its mean value in a given spectral bin. The random noise component $\mathrm{NEdN}_{\mathrm{r}}$ in our approach is 
estimated applying a standard PCA technique to the same data set of BB measurements. For this analysis a set of $\mathrm{M}=300-900$ interferograms of ECT, ICT, or DS blackbodies is collected for each interferometer sweep and then these interferograms are radiometrically and spectrally calibrated using the standard SDR algorithm.

The PCA procedure of random noise estimation includes several steps ${ }^{[8]}$ :

1. Normalize each difference of observed spectra $\mathrm{R}_{\mathrm{i}, \mathrm{j}}(\nu, \mathrm{m})$ with its mean value $\bar{R}_{i}$ by the estimated instrument

noise:

$$
\Delta \mathbf{R}=\left|\frac{R_{i j}-\bar{R}_{i}}{N E d N_{j}}\right|
$$

Where $v$ is a wavenumber of $\mathrm{i}$-channel and $\mathrm{m}=1 \ldots \mathrm{M}$ is an observation number.

2. Generate the covariance matrix of the observations: $\quad \mathbf{C}=\frac{1}{M-1} \cdot \Delta \mathbf{R}^{\mathrm{T}} \times \Delta \mathbf{R}$

3. Derive the principal components (PCs) of the covariance matrix: $\mathbf{C}=\mathbf{U} \times \boldsymbol{\Lambda} \times \mathbf{U}^{\mathbf{T}}$

Where $\mathbf{U}$ is a matrix of eigenvectors and $\boldsymbol{\Lambda}$ is a matrix of eigenvalues.

4. Determine optimal number $\boldsymbol{t}$ of PCs to reconstruct the signal

5. Project each spectra onto PCs:

$$
\mathbf{P}=\Delta \mathbf{R} \times \mathbf{U}
$$

6. Reconstruct noise free signal retaining only the first most significant $\boldsymbol{t}$-PCs: $\Delta \mathbf{R}^{r e c}=\mathbf{P}_{\mathbf{t}} \times \mathbf{U}_{\mathbf{t}}^{T}$

7. To return to the radiance space, multiply by the noise estimate and add mean radiance $\bar{R}_{i}$

Random noise, filtered out after reconstruction of the original data by truncated set of optimal number of PCs, can be estimated as the standard deviation of the residual errors between the original and reconstructed data:

$$
N E d N_{r}\left(v_{i}\right)=\sqrt{\frac{1}{M-1} \sum_{J=1}^{M}\left(R_{i j}^{r e c}-R_{i j}^{o b s}\right)^{2}}
$$

Knowing the total noise and its random component, the correlated component $\mathrm{NEdN}_{\mathrm{c}}$ can be easily estimated according to the equation (1). In our experience, only one PC needs to be retained to reconstruct typical BB spectra when random noise is dominant or comparable with spectrally correlated noise. More PCs (typically 2-4) are needed for optimal reconstruction of the observed BB spectra when spectrally correlated noise dominates total $\mathrm{NEdN}_{\mathrm{t}}$.
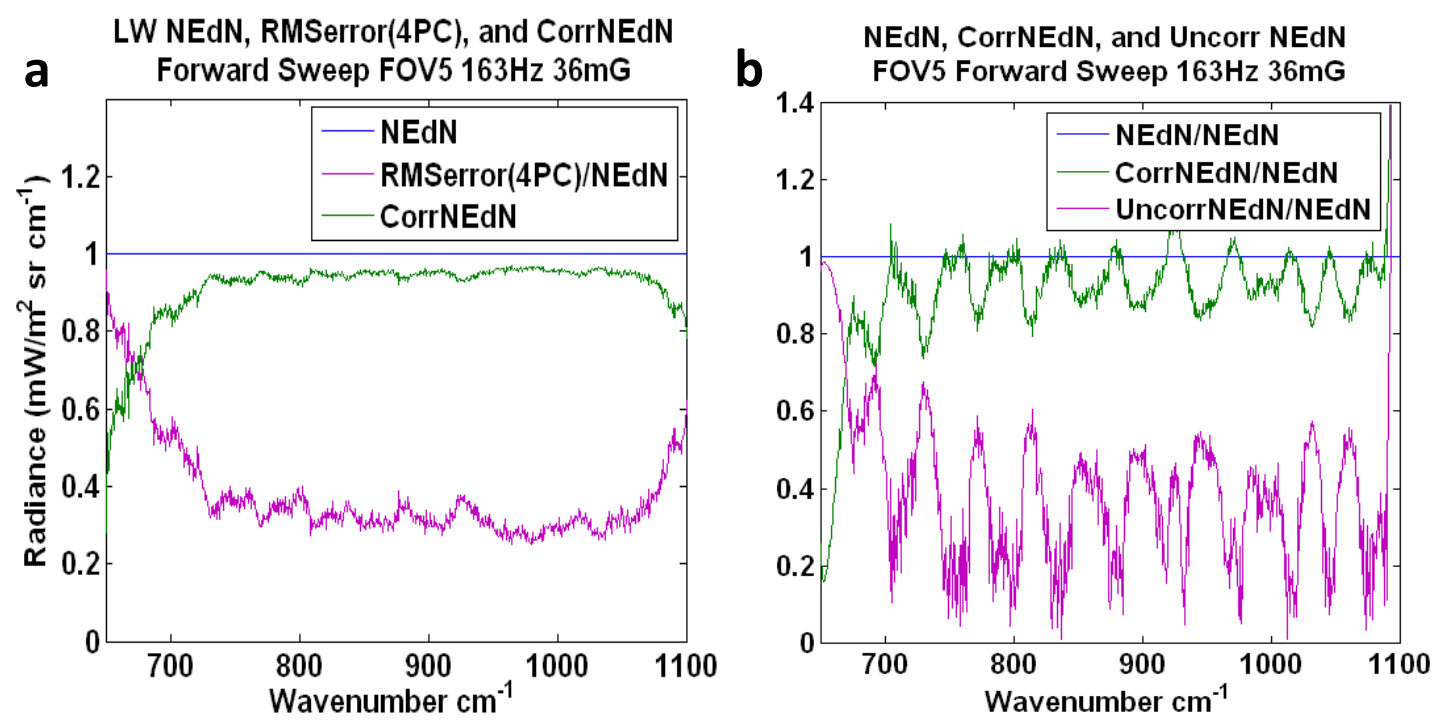

Figure 11. Contribution of random (magenta) and correlated (green) noise components to the total NEdN (blue) estimated during vibration test using PCA (a) and Antonelli et al. ${ }^{[8]}$ (b) approaches. Data is normalized by the total NEdN. 
An alternative method for estimating the random and correlated noise components using a set of BB spectra was proposed by Antonelli et. al ${ }^{[8]}$. In their approach, the random and correlated noise components are estimated using set of noise variances obtained on the original data and on the same data set spectrally averaged within a running window of $50 \mathrm{~cm}^{-1}$. In general, both approaches give practically the same results on the set of BB spectra when random noise dominates or is comparable with the correlated noise. However, some differences are observed when correlated noise component dominates. In Figure 11, the results of these two approaches are compared for the case of dominant correlated noise. The data was acquired under external vibration at $163 \mathrm{~Hz}$ with an acceleration level of $36 \mathrm{mg}$ applied to the instrument during the CrIS dynamic interaction test.

Both methods show that correlated noise dominates and produces approximately the same noise level. However, the PCA method produces consistent results over the whole spectral band while the Antonelli et.al. ${ }^{[8]}$ method leads to the artificial oscillations most probably induced by the running window. This example outlines the main advantage of the PCA technique. The PCA approach captures spectral correlation over the whole spectral band under analysis while the alternative approach captures only correlation within the averaging running spectral window $\left(50 \mathrm{~cm}^{-1}\right.$ in this case).

\section{REFERENCES}

[1] Glumb, R.J., Williams, F.L., Funk, N., Chateauneuf, F., Roney, A., and Allard, R., “Cross-track Infrared Sounder (CrIS) development status," Proc. SPIE 5152, 1-8 (2003).

[2] Aumann, H.H.,. Chahine, M.T, Gautier, C., Goldberg, M.D., Kalnay, E., McMillin, L.M., Revercomb, H., Rosenkranz, P.W., Smith, W.L., Staelin, D.H., Strow, L.L., and Susskind, J., "AIRS/AMSU/HSB on the Aqua Mission: Design, science objectives, data products, and processing systems," IEEE Trans. on Geosc. Rem. Sensing 41, 410-417 (2003).

[3] Blumstein, D., Chalon, G., Carlier, T., Buil, C., Hebert, P., Maciaszek, T., Ponce, G., and Phulpin, T., "IASI instrument: technical overview and measured performances," Proc. SPIE 5543, 196-207 (2004).

[4] Klaes, K. D., Cohen, M., Buhler, Y., Schluessel, P., Munro, P., Luntama,J.-P., Von Engeln, A., Clerigh, E. O., Bonekamp, H., Ackernmann, J., and Schmetz, J., "An introduction to the EUMETSAT polar system," B. Am. Meteorol. Soc. 88, 1085-1096 (2007).

[5] NPOESS Integrated Program Office, "Cross-track Infrared Sounder (CrIS) sensor data record algorithm theoretical basis document (ATBD)," Doc. \# D43773, Revision D (2009).

[6] Taylor, J. K., Tobin, D.C., Revercomb, H. E., Knutesen, R. O., Borg, L., Best, F. A., “Analysis of CrIS flight model 1 radiometric linearity," OSA FTS Topical Meeting 2009, 26-30 ( 2009).

[7] D.C. Tobin, L. Borg, J. K. Taylor, R. O. Knutesen, F. A. Best, H.E. Revercomb, "Infrared Spectral Radiance Validation and plans for the cross-track Infrared Sounder (CrIS)", OSA FTS Topical meting, Vancouver, BC, Canada, 26-30 April (2009).

[8] Antonelli P., H. E. Revercomb, and L. A. Sromovsky, "A principle component noise filter for high spectral resolution infrared measurements”, J. Geophys. Res. 109, D23102, doi:10.1029/2004JD004862 (2004).

[9] David Tobin, Henry Revercomb, Paolo Antonelli, Kenneth Vinson, Steven Dutcher, Robert Knuteson, Joseph Taylor, Fred Best, Chris Moeller, Mathew Gunshor, "Recent efforts to validate EOS observations. Hyperspectral data noise characterization using PCA: application to AIRS", Proc. SPIE Optics and Photonics V. 6301, pp 630107, San Diego, CA (2006).

[10] Gail E. Bingham, Chad Fish, Vladimir V. Zavyalov, Christopher D. Barnet, Dave Tobin, Larrabee Strow and Denise Hagan, "The NPOESS Preparatory Project (NPP) Cross-track Infrared Scanner (CrIS) predicted sensor performance calibration and preliminary data product performance", IGARSS MO3.09.5, July 12-17, Cape Town, South Africa (2009). 